PROCEEDINGS OF THE

AMERICAN MATHEMATICAL SOCIETY

Volume 127, Number 9, Pages 2767-2772

S 0002-9939(99)04843-1

Article electronically published on April 15, 1999

\title{
VARIATIONAL PRINCIPLES FOR AVERAGE EXIT TIME MOMENTS FOR DIFFUSIONS IN EUCLIDEAN SPACE
}

\author{
KIMBERLY K. J. KINATEDER AND PATRICK MCDONALD
}

(Communicated by Stanley Sawyer)

\begin{abstract}
Let $D$ be a smoothly bounded domain in Euclidean space and let $X_{t}$ be a diffusion in Euclidean space. For a class of diffusions, we develop variational principles which characterize the average of the moments of the exit time from $D$ of a particle driven by $X_{t}$, where the average is taken over all starting points in $D$.
\end{abstract}

\section{INTRODUCTION}

In this note we study diffusions on $\mathbb{R}^{d}$ and properties of their corresponding exit times from smoothly bounded, connected, open domains in $\mathbb{R}^{d}$ with compact closure. We will denote by $X_{t}$ a diffusion in $\mathbb{R}^{d}$ with corresponding generator $L$ a uniformly elliptic operator of divergence form. We will write $L f=\operatorname{div}(a \nabla f)$ where the coefficient matrix $a=a_{i j}(x)$ is smooth and symmetric.

Let $\tau=\tau(\omega)=\inf \left\{t \geq 0: X_{t}(\omega) \notin D\right\}$ be the first exit time of $X_{t}$ from $D(S)$.

We study the average $k$ th moment of the exit time for a particle driven by $X_{t}$, starting in $D$ :

$$
\mathcal{E}_{k}=\mathcal{E}_{k}(D)=\int_{D} E_{x}\left(\tau^{k}\right) d x
$$

where $E_{x}$ denotes expectation under the measure $P_{x}$ satisfying $P_{x}\left\{X_{0}=x\right\}=1$, for all $x \in \mathbb{R}^{d}$. Note that $\mathcal{E}_{k}$ is invariant under Euclidean motions.

We give a variational characterization of $\mathcal{E}_{k}$ for each positive integer value of $k$ in the following theorem:

Theorem 1.1. Let $X_{t}$ be a diffusion on $\mathbb{R}^{d}$ with generator $L$ a uniformly elliptic operator of divergence form, $L f=\operatorname{div}(a \nabla f)$, where the coefficient matrix a is smooth and symmetric. Let $D$ be a smoothly bounded open domain in $\mathbb{R}^{d}$ with compact closure, $\bar{D}$. Define $\mathcal{E}_{k}$ as above and let $\mathcal{F}_{k}$ be defined by

$$
\mathcal{F}_{k}=\left\{f \in C^{\infty}(\bar{D}) ; \int_{D} f(x) d x \neq 0, f=L f=\cdots=L^{k-1} f=0 \text { on } \partial D\right\} .
$$

Received by the editors June 2, 1997 and, in revised form, November 24, 1997.

1991 Mathematics Subject Classification. Primary 60J65, 58G32.

Key words and phrases. Diffusions, exit times, variational principles.

The second author was partially supported by a DSR grant from the University of South Florida.

(C)1999 American Mathematical Society 
Let $\llbracket \frac{k}{2} \rrbracket$ be the greatest integer of $\frac{k}{2}$. Then, for $k$ even,

$$
\mathcal{E}_{k}=k ! \sup _{f \in \mathcal{F}_{k}} \frac{\left(\int_{D} f\right)^{2}}{\int_{D}\left|L^{\frac{k}{2}} f\right|^{2}}
$$

and for $k$ odd,

$$
\mathcal{E}_{k}=k ! \sup _{f \in \mathcal{F}_{k}} \frac{\left(\int_{D} f\right)^{2}}{\int_{D}\left|\nabla L \llbracket \frac{k}{2} \rrbracket f\right|_{L}^{2}}
$$

where $\langle\nabla f, \nabla g\rangle_{L}=\sum_{i, j} a_{i j} \frac{\partial f}{\partial x_{j}} \frac{\partial g}{\partial x_{i}}$ is the inner product associated with $L$.

The proof of Theorem 1.1 is an application of the generalized Dynkin formula [AK] (cf. also [P1]), followed by an explicit computation. That smooth minimizers for the variational principles cited in Theorem 1.1 exist is explicit in our computations.

Our study of the sequence $\left\{\mathcal{E}_{k}\right\}$ is largely motivated by the now classic work in spectral analysis concerning to what extent a smoothly bounded domain in Euclidean space is determined by its Dirichlet spectrum. More precisely, when the diffusion is standard Brownian motion with generator $L=\frac{1}{2} \Delta$, we are interested in studying to what extent the sequence $\left\{\mathcal{E}_{k}\right\}$ determines the geometry of the underlying domain. There are a number of preliminary results in this direction. For example, in $[\mathrm{KMM}]$ the authors prove that among domains of a fixed volume, each element of the sequence is maximized if and only if the underlying domain is a ball of the appropriate volume.

For the case $k=1$, it is known that the functional $\mathcal{E}_{1}$ computes the torsional rigidity of a domain. The St. Venant torsion problem, a problem with a long and distinguished history, is to determine those domains of a given volume which maximize torsional rigidity. The problem was settled by Polya (cf. [P2]) who proved that among domains of a fixed volume, the torsional rigidity is maximized by a ball. This result can be recovered using (1.2) and properties of the quotient given in (1.2) under symmetric rearrangement (cf. also [KM1]).

\section{BASIC RESULTS AND DEFINITIONS}

Let $(\Omega, \mathcal{B})$ be a measurable space and $\left\{P_{x}\right\}_{x \in \mathbb{R}^{d}}$ a family of probability measures on $(\Omega, \mathcal{B})$. Let $\left\{X_{t}\right\}_{t \geq 0}$ denote a $d$-dimensional diffusion with generator $L$, a uniformly elliptic operator in divergence form and for which $P_{x}\left\{X_{0}=x\right\}=1$, for $x \in \mathbb{R}^{d}$.

Let $D$ be a smoothly bounded, connected, open domain with compact closure. As in the introduction, we define the first exit time for a particle driven by $X_{t}$ from $D$ by $\tau=\tau(\omega)=\inf \left\{t: X_{t}(\omega) \notin D\right\}$. For each $x \in \mathbb{R}^{d}$, we will denote the expected value of a random variable $Y$ under the probability measure $P_{x}$ by $E_{x}(Y)$.

There is a useful relationship between the solution of a certain Poisson problem on the domain $D$ and the expected value of the $k$ th power of the first exit time of a particle driven by $X_{t}$ from $D$ starting at $x \in D$. Suppose $u_{k}$ solves the problem

$$
\begin{aligned}
L^{k} u_{k}+(-1)^{k-1} k ! & =0 \text { on } D, \\
u_{k}=L u_{k}=\cdots L^{k-1} u_{k} & =0 \text { on } \partial D .
\end{aligned}
$$


Note that $u_{k}$ can be defined inductively by

$$
\begin{aligned}
L u_{1}+1 & =0 \text { on } D, \\
u_{1} & =0 \text { on } \partial D
\end{aligned}
$$

and

$$
\begin{aligned}
L u_{k}+k u_{k-1} & =0 \text { on } D, \\
u_{k} & =0 \text { on } \partial D .
\end{aligned}
$$

Using the generalized Dynkin formula $[\mathrm{H}]$ (cf. also $[\mathrm{AK}]$ and $[\mathrm{P} 1]$ ) we have

$$
\begin{aligned}
E_{x}\left[u_{k}\left(X_{0}\right)\right]-E_{x}\left[u_{k}\left(X_{\tau}\right)\right] & =\sum_{j=1}^{k-1} \frac{(-1)^{j}}{j !} E_{x}\left[\tau^{j} L^{j} u_{k}\left(X_{\tau}\right)\right] \\
& +\frac{(-1)^{k}}{(k-1) !} E_{x}\left[\int_{0}^{\tau} s^{k-1} L^{k} u_{k}\left(X_{s}\right) d s\right] .
\end{aligned}
$$

Using the definition of $u_{k}$ and $\tau$, this gives $u_{k}(x)=E_{x}\left[\tau^{k}\right]$ and $\mathcal{E}_{k}$ can be expressed in terms of $u_{k}$ by $\mathcal{E}_{k}(D)=\int_{D} u_{k}(x) d x$.

We will need a number of integral formulae involving the function $u_{1}$ and the geometry of the diffusion $L$. To ease notation in the sequel we define, for $\alpha$ and $\beta$ tangent vectors at $x \in D$, a scalar product, $\langle\alpha, \beta\rangle_{L}$, by

$$
\langle\alpha, \beta\rangle_{L}=\alpha^{T} a(x) \beta
$$

where $\alpha^{T}$ denotes the transpose of $\alpha$.

Let $u_{1}$ be as defined in (2.1) and let $f \in \mathcal{F}_{k}$. Let $\nu$ be the outward pointing unit normal vector to $\partial D$. By the Divergence Theorem,

$$
\int_{D} f L u_{1}-u_{1} L f=\int_{\partial D} f\left\langle\nabla u_{1}, \nu\right\rangle_{L}-u_{1}\langle\nabla f, \nu\rangle_{L}=0 .
$$

We conclude

$$
\int_{D} f=-\int_{D} u_{1} L f
$$

If $X$ is a vectorfield on $D$ and $f \in \mathcal{F}_{k}$, then $\operatorname{div}(f X)=f \operatorname{div}(X)+\langle\nabla f, X\rangle$ where $\langle\alpha, \beta\rangle$ is the standard scalar product. By the Divergence Theorem,

$$
\int_{D} \operatorname{div}(f X)=\int_{\partial D} f\langle X, \nu\rangle=0
$$

and we conclude that

$$
\int_{D} f \operatorname{div}(X)=-\int_{D}\langle\nabla f, X\rangle .
$$

In particular, if $u_{1}$ is as defined in (2.1) and $X=a_{i j}(x) \nabla u_{1}$, then

$$
\int_{D} f=\int_{D}\left\langle\nabla u_{1}, \nabla f\right\rangle_{L}
$$




\section{VARIATIONAL CHARACTERIZATIONS}

Throughout this section let $D$ be as above and let $\mathcal{F}_{k}$ be given as in Theorem 1.1. We begin with a lemma which generalizes (2.4) and (2.5).

Lemma 3.1. Let $u_{n}$ be as defined by (2.2), let $k$ be a positive integer, and let $f \in \mathcal{F}_{k}$. If $k=2 n$, then

$$
\int_{D} f=\frac{(-1)^{n}}{n !} \int_{D} u_{n} L^{n} f
$$

If $k=2 n+1$, then

$$
\int_{D} f=\frac{(-1)^{n}}{(n+1) !} \int_{D}\left\langle\nabla u_{n+1}, \nabla L^{n} f\right\rangle_{L}
$$

where the scalar product is as given in (2.3).

Proof. Suppose that $k=2 n$ and for $0 \leq l \leq n-1$, define

$$
P_{l}=\left(L^{l} u_{n}\right)\left(L^{n-l} f\right)-\left(L^{l+1} u_{n}\right)\left(L^{n-(l+1)} f\right) .
$$

Then

$$
\sum_{l=0}^{n-1} P_{l}=u_{n} L^{n} f-f L^{n} u_{n}
$$

Let $\nu$ be the outward pointing unit normal vector along $\partial D$. By the Divergence Theorem and the fact that $L^{l} u_{n}=0$ on $\partial D$, for $l=0, \ldots, n-1$,

$$
\int_{D} P_{l}=\int_{\partial D}\left(L^{l} u_{n}\right)\left\langle\nabla L^{n-l-1} f, \nu\right\rangle_{L}-\left(L^{n-(l+1)} f\right)\left\langle\nabla L^{l} u_{n}, \nu\right\rangle_{L}=0 .
$$

Combining (3.3) and (3.4) and using that $L^{n} u_{n}=(-1)^{n} n$ !, we have established (3.1).

Suppose $k=2 n+1$ and for $0 \leq l \leq n$, define

$$
R_{l}=\left(L^{l} u_{n+1}\right)\left(L^{n+1-l} f\right)-\left(L^{l+1} u_{n+1}\right)\left(L^{n+1-(l+1)} f\right) .
$$

Then

$$
\sum_{l=0}^{n} R_{l}=u_{n+1} L^{n+1} f-f L^{n+1} u_{n+1} .
$$

As above, we use the Divergence Theorem to see that

$$
\int_{D} R_{l}=\int_{\partial D}\left(L^{l} u_{n+1}\right)\left\langle\nabla L^{n-l} f, \nu\right\rangle_{L}-\left(L^{n-l} f\right)\left\langle\nabla L^{l} u_{n+1}, \nu\right\rangle_{L}=0 .
$$

Since $L^{n+1} u_{n+1}=(-1)^{n+1}(n+1)$ !, we conclude

$$
\int_{D} f=\frac{(-1)^{n+1}}{(n+1) !} \int_{D} u_{n+1} L\left(L^{n} f\right)
$$

If $X$ is the vectorfield given by $X=a \nabla\left(L^{n} f\right)$, then following the argument used to establish (2.5),

$$
\begin{aligned}
\int_{D} u_{n+1} L\left(L^{n} f\right) & =\int_{D} u_{n+1} \operatorname{div}(X) \\
& =-\int_{D}\left\langle\nabla u_{n+1}, \nabla L^{n} f\right\rangle_{L}
\end{aligned}
$$

and we have established (3.2). 
We now prove Theorem 1.1. Suppose $k=2 n$ and, for $f \in \mathcal{F}_{k}$, consider the quotient

$$
Q_{k}(f)=\frac{\left(\int_{D} f\right)^{2}}{\int_{D}\left|L^{n} f\right|^{2}}
$$

From (3.1)

$$
Q_{k}(f)=\frac{\left(\frac{1}{n !}\right)^{2}\left(\int_{D} u_{n} L^{n} f\right)^{2}}{\int_{D}\left|L^{n} f\right|^{2}}
$$

Let $\mathcal{G}_{k}=\left\{g \in \mathcal{F}_{n}: g=L^{n} f\right.$ for some $\left.f \in \mathcal{F}_{k}\right\}$. Let $\mathcal{H}_{k}$ be the completion of $\mathcal{G}_{k}$ in the Hilbert space, $\mathcal{L}^{2}$, of square integrable functions on $D$. If we denote the inner product of $g$ and $h \in \mathcal{L}^{2}$ by $\langle g, h\rangle$ and by $\|g\|$ the $\mathcal{L}^{2}$ norm of $g$, then we can view $Q_{k}$ as a map $Q_{k}: \mathcal{G}_{k} \subset \mathcal{H}_{k} \rightarrow \mathbb{R}$,

$$
Q_{k}(g)=\left(\frac{1}{n !}\right)^{2}\left(\frac{\left\langle u_{n}, g\right\rangle}{\|g\|}\right)^{2} .
$$

Clearly, the domain of $Q_{k}$ can be extended to nonzero elements of $\mathcal{H}_{k}$ and $Q_{k}(\mathrm{cg})=$ $Q_{k}(g)$ for every nonzero scalar $c$. It follows that $Q_{k}$ is maximized when $g \in \mathcal{H}_{k}$ is in the direction of $u_{n} \in \mathcal{H}_{k}$. If $g=c u_{n}$ we have $L^{n}\left(c^{\prime} u_{2 n}\right)=g$, and computing $Q_{k}\left(c u_{n}\right)$ we see that

$$
\begin{aligned}
\sup _{g \in \mathcal{H}_{k}} Q_{k}(g) & =Q_{k}\left(c u_{n}\right) \\
& =\frac{\left(\int_{D} u_{2 n}\right)^{2}}{\int_{D}\left(L^{n} u_{2 n}\right)^{2}}
\end{aligned}
$$

where we have applied (3.1) of Lemma 3.1 to the numerator. Note that $\left(L^{n} u_{2 n}\right)^{2}=$ $\frac{(-1)^{n}}{n !}(2 n) ! u_{n} L u_{2 n}$. Applying Lemma 3.1 to the denominator we obtain

$$
\begin{aligned}
\sup _{g \in \mathcal{H}_{k}} Q_{k}(g) & =\frac{\left(\int_{D} u_{2 n}\right)^{2}}{(2 n) ! \int_{D} u_{2 n}} \\
& =\frac{1}{k !} \mathcal{E}_{k}(D)
\end{aligned}
$$

which establishes (1.1) of Theorem 1.1.

The proof of (1.2) of Theorem 1.1 is similar. Suppose $k=2 n+1$ and, for $f \in \mathcal{F}_{k}$, consider the quotient

$$
\tilde{Q}_{k}(f)=\frac{\left(\int_{D} f\right)^{2}}{\int_{D}\left|\nabla L^{n} f\right|_{L}^{2}} .
$$

From (3.2) of Lemma 3.1,

$$
\tilde{Q}_{k}(f)=\frac{\left(\frac{1}{(n+1) !}\right)^{2}\left(\int_{D}\left\langle\nabla u_{n+1}, \nabla L^{n} f\right\rangle_{L}\right)^{2}}{\int_{D}\left|\nabla L^{n} f\right|_{L}^{2}} .
$$

Let $C^{\infty}\left(\bar{D}, \mathbb{R}^{d}\right)$ be the space of smooth vectorfields on $\bar{D}$. Let

$$
\tilde{\mathcal{G}}_{k}=\left\{X \in C^{\infty}\left(\bar{D}, \mathbb{R}^{d}\right): X=\nabla g \text { for some } g \in \mathcal{F}_{n+1}\right.
$$

with $g=L^{n} f$ for some $\left.f \in \mathcal{F}_{k}\right\}$. 
Let $\tilde{\mathcal{H}}_{k}$ be the completion of $\tilde{\mathcal{G}}_{k}$ in the space of vectorfields square integrable with respect to the inner product $\langle\alpha, \beta\rangle_{L}$. We can view $\tilde{Q}_{k}$ as a map $\tilde{Q}_{k}: \tilde{\mathcal{G}}_{k} \subset \tilde{\mathcal{H}}_{k} \rightarrow \mathbb{R}$,

$$
\tilde{Q}_{k}(g)=\left(\frac{1}{(n+1) !}\right)^{2}\left(\frac{\left\langle\nabla u_{n+1}, g\right\rangle_{L}}{\|g\|_{L}}\right)^{2} .
$$

It is clear that the domain of $\tilde{Q}_{k}$ extends to nonzero vectors in the space $\tilde{\mathcal{H}}_{k}$ and that for all nonzero scalars $c, \tilde{Q}_{k}(c g)=\tilde{Q}_{k}(g)$. It follows that $\tilde{Q}_{k}$ is maximized when $g=c \nabla u_{n+1}$ where $c$ is some nonzero constant. Computing $\tilde{Q}_{k}\left(\nabla u_{n+1}\right)$ we see that

$$
\begin{aligned}
\sup _{g \in \tilde{\mathcal{H}}_{k}} \tilde{Q}_{k}(g) & =\tilde{Q}_{k}\left(c \nabla u_{n+1}\right) \\
& =\frac{\left(\int_{D} u_{2 n+1}\right)^{2}}{\int_{D}\left\|\nabla L^{n} u_{2 n+1}\right\|_{L}^{2}}
\end{aligned}
$$

where we have used (3.2) on the numerator.

Note that $\left\|\nabla L^{n} u_{2 n+1}\right\|_{L}^{2}=\frac{(-1)^{n}}{(n+1) !}(2 n+1) !\left\langle\nabla u_{n+1}, \nabla L^{n} u_{2 n+1}\right\rangle_{L}$. Applying (3.2) of Lemma 3.1 to the denominator we obtain

$$
\begin{aligned}
\sup _{g \in \tilde{\mathcal{H}}_{k}} \tilde{Q}_{k}(g) & =\frac{\left(\int_{D} u_{2 n+1}\right)^{2}}{(2 n+1) ! \int_{D} u_{2 n+1}} \\
& =\frac{1}{k !} \mathcal{E}_{k}(D)
\end{aligned}
$$

which establishes (1.2) of Theorem 1.1.

\section{REFERENCES}

[AK] Athreya, K. B. and Kurtz, T. G. (1973), A generalization of Dynkin's identity, Ann. of Prob. 1, 570-579. MR 50:1342

[H] Ha'sminskii, R. Z. (1960), Probabilistic representation of the solution of some differential equations, Proc. 6th All Union Conf. on Theor. Probability and Math. Statist. (Vilnius 1960) (See Math Rev. 3127 v.32 (1966) pg. 632). MR 32:3127

[KM1] Kinateder, K. K. J. and McDonald, P. (1997), Brownian functionals on hypersurfaces in Euclidean space, Proc. Amer. Math. Soc. 125 6, 1815-1822. MR 97g:60109

[KMM] Kinateder, K. K. J., McDonald, P., and Miller, D. F., Boundary Value Problems and Exit Times for Diffusions in Euclidean Space. Prob. Th. Rel. 111 (1998), pp. 469-487. CMP 99:01

[P1] Pinsky, M., Mean exit times and hitting probabilities of Brownian motion in geodesic balls and tubular neighborhoods, Stochastic Processes - Mathematics and Physics, Lecture Notes in Mathematics, vol. 1158, Springer Verlag, New York, pp. 216-223. MR 87g:58129

[P2] Polya, G. (1948), Torsional rigidity, principal frequency, electrostatic capacity and symmetrization, Quart. Appl. Math. 6, 267-277. MR 10:206b

Department of Mathematics, Wright State University, Dayton, Ohio 45435

Department of Mathematics, New College of the University of South Florida, SaraSOTA, FLORIDA 34243

E-mail address: pmacdona@virtu.sar.usf.edu 